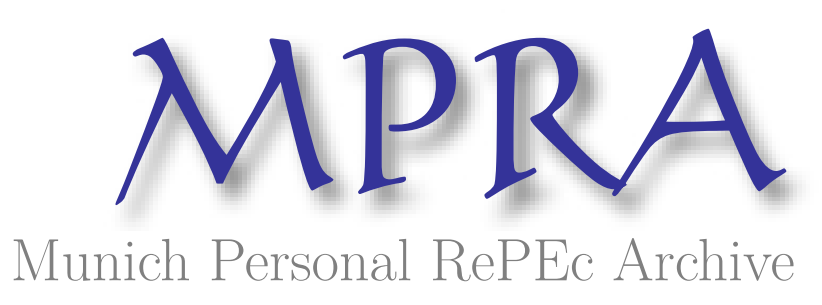

\title{
Using Count Data Models in Travel Cost Analysis with Aggregate Data
}

Hellerstein, Daniel

Economic Research Service, USDA

1991

Online at https://mpra.ub.uni-muenchen.de/25264/

MPRA Paper No. 25264, posted 23 Sep 2010 14:03 UTC 


\title{
Using Count Data Models in Travel Cost Analysis with Aggregate Data
}

\author{
Daniel M. Hellerstein
}

\begin{abstract}
In order to control for censoring and the integer nature of trip demand, the use of count data models in travel cost analysis is attractive. Two such models, the Poisson and negative binomial, are discussed. Robust estimation techniques that loosen potentially stringent distributional assumptions are also reviewed. For illustrative purposes, several count data models are used to estimate a county-level travel cost model using permit data from the Boundary Waters Canoe Area.
\end{abstract}

Key words: Boundary Waters Canoe Area, count data, negative binomial, Poisson, travel cost.

Estimators of recreational demand models frequently use continuous functional forms, such as ordinary least squares (OLS) on log transformed variables (e.g., Ziemer, Musser, and Hill). However, the nature of trip demand introduces complicating factors. First, trips occur in nonnegative quantities. Failure to control for this censoring will lead to biased estimation. Second, because trips are available only in integer quantities, the usual demand models, which correlate marginal quantity with marginal price, may be inapplicable.

In light of these factors, a natural alternative is to use statistical models that explicitly recognize the "count" nature of trip demand. Several recent papers (e.g., Shaw, Smith, Grogger and Carson, Creel and Loomis) have applied count models to the travel cost model. These works largely have focused on truncated data sets based on choice-based samples. In this study the focus is on the older problem where zero-demanders are included. In particular, the application of several robust estimators of count models to aggregated data will be considered.

The Poisson distribution forms the foundation for the count models examined in this study. Although the Poisson is a convenient distribution

\footnotetext{
Daniel M. Hellerstein is a natural resource economist with the Resources and Technology Division, Economic Research Service, U.S. Department of Agriculture.

Portions of this research were supported by the U.S. Forest Service Rocky Mountain Forest and Range Experiment Station.

The views expressed here are not necessarily those of either the Forest Service or the Economic Research Service.

The author acknowledges Robert Meldelsohn, Michael Moore, George Peterson, and A. Colin Cameron for their advice and assistance.
}

to work with, it imposes some stringent constraints on the demand distribution. In particular, the Poisson distribution assumes the variance of trip demand is equal to the expected value of trip demand. To loosen these constraints, a generalization of the Poisson, the negative binomial, is discussed. Robust estimation procedures, that permit further loosening of a priori assumptions are then reviewed. Permit data from the Boundary Waters Canoe Area are used to examine the effects of these count models on consumer surplus estimates and on coefficient variability.

\section{Theory}

In formulating a demand process that yields count data, one must consider that trips are not available in continuous quantities. The integer nature of the data can be explicitly accounted for by modeling the observed number of trips taken (over a season) as the result of many discrete choices (say, one for each day of the season). Under this scenario, count data distributions, such as the Poisson, are an asymptotic outcome.'

Therefore, in estimating a count model, the analyst is implicitly estimating the "daily" probability of the recreator choosing to visit. Increasing the travel cost will reduce the probability of a visit on any given day. Following Small

\footnotetext{
' If the probability of taking a trip on any given day is small, constant and independent of earlier decisions, and if the number of days in a season is large, then the distribution of trips will approach a Poisson process.
} 
and Rosen, integrating over these price changes yields a measure of the compensating variation. Extending these results to the repeated discrete choice context yields a consumer surplus measure over an entire season (Hellerstein and Mendelsohn). The key result is that count data models, as the limit of a repeated discrete choice process, can be used much like continuous models. In particular, integrating under a continuous estimator of predicted demand will yield a measure of consumer surplus.

Given this background, we concentrate on estimating the expected value of trip demand. Furthermore, as a result of repeated discrete choice, the number of observed trips will follow a Poisson distribution. Formally, the expected value of demand is

$$
E(Y)=f(P, Z ; \beta),
$$

where $E(Y)$ is the expected number of trips taken per season, $P, Z$ are explanatory variables including travel cost to site $(P)$ and demand shift variables $(Z)$, such as income and travel costs to substitute sites, and $\beta$ is a vector of coefficients.

The Poisson probability distribution of demand is

$$
\operatorname{Prob}(Y=n ; n=0,1,2, \ldots)=\exp (-\lambda) \lambda^{n} / n \text { ! }
$$

with $\lambda=f(\mathrm{P}, Z ; \beta)$.

The Poisson is a single parameter distribution with expectation and variance both equal to $\lambda$ (Mood, Graybill, and Boes). Although $n$ is a nonnegative integer, $\lambda$ must be a strictly positive real number.

The Poisson model is solved by estimating $\beta$, say $\beta^{*}$, in $\lambda^{*}=\lambda\left(P, Z ; \beta^{*}\right)$. The estimated value of $\lambda, \lambda^{*}$, is interpreted as the predicted expected value (and variance) of demand. The predicted expected value of consumer surplus, $E(C S)$, is then computed via the usual integration:

$$
E(C S)=\int_{P_{o b s}}^{P_{\max }} \lambda\left(P, Z ; \beta^{*}\right) d P,
$$

where $P_{o b s}$ is observed price, and $P_{\max }$ is a choke price, possibly $\infty$.

This paper will focus on this measure of consumer surplus estimated using the predicted mean of demand. Criticisms of this approach in the continuous context (see Bockstael and Strand; or Adamowicz, Fletcher, Graham-Tomasi) stress the importance of considering the source of error. However, if measurement error is small, and if the measure of expected demand is unbiased, then this measure of consumer surplus will be a good approximation to the expected value of consumer surplus. ${ }^{2}$

\section{Extending the Poisson: The Negative Binomial}

A drawback to the Poisson model is the implied assumption that $E(Y)$ and $\sigma^{2}(Y)$ are equal. Furthermore, Poisson "regressions" allow no random component in the $\lambda$ estimator; the $\lambda=\lambda(P$, $\left.Z ; \beta^{*}\right)$ relationship does not contain an error component.

The negative binomial count model is often used to relax this unlikely condition of perfect knowledge of the $\lambda$ estimator and to permit more flexible variance/mean relationships. Following Cameron and Trivedi, the negative binomial is derived as a compound Poisson distribution, where $\lambda$ is assumed to be distributed as a gamma random variable. ${ }^{3}$ Integrating over this distribution of $\lambda$ yields the two parameter negative binomial. Formally,

$$
\begin{aligned}
\operatorname{Prob}(Y= & n, n=0,1, \ldots) \\
& =\frac{\Gamma(n+\nu)}{\Gamma(n+1) \Gamma(\nu)}\left(\frac{\nu}{\nu+\mu}\right)^{\nu}\left(\frac{\mu}{\nu+\mu}\right)^{n},
\end{aligned}
$$

with

$$
E(Y)=\mu \text { and } \sigma^{2}(Y)=\mu+\frac{\mu^{2}}{\nu} .
$$

The variance to mean ratio of the negative binomial is a decreasing function of $\nu$. As $\nu$ approaches infinity, the negative binomial collapses to the Poisson; hence the Poisson is nested within the negative binomial.

In terms of the repeated discrete choice framework, the negative binomial admits that

\footnotetext{
${ }^{2}$ The concern is that $E(C S(Y))$ may not equal the $C S(E(Y))$, where the latter is what the analyst computes. However,

$$
\int_{-\infty}^{\infty} \int_{P_{0}}^{P_{1}} d(x ; \beta, \epsilon) d P d F(\epsilon)=\int_{P_{0}}^{P_{1}} d^{*}(x ; \beta) d P
$$

with $d^{*}=E(Y)$. Therefore, as long as $E(Y)$ is unbiased, then $E(C S(Y))$, the left-hand side of the above equation, will approach $C S(E(Y))$, the right-hand side. It must be stressed that $d^{*}$ is an unbiased estimate of the expected value of demand. In particular, $d^{*}$ is assumed to explicitly account for censoring. When this constraint cannot be guaranteed, an alternative form for $d^{*}=E(Y)$ (such as suggested by Maddala, p. 158) should be used.

${ }^{3}$ An alternative derivation can also be found in Hausman, Hall, and Griliches. The Cameron and Trivedi formulation is more flexible, including the Hausman, Hall, and Griliches specification as a special case.
} 
the underlying daily probability of visiting may be randomly distributed. More concisely, each individual is assumed to draw a value for her daily probability at the beginning of the season. Knowledge of the random process generating these daily probabilities is not required, so long as the net result is a gamma distribution of $\lambda$, conditional on the exogenous variables.

\section{Estimation}

In addition to their appealing statistical properties, the Poisson and negative binomial have several useful empirical properties:

(a) The sum of $W$ independent Poisson variates is also Poisson distributed, with parameter $\lambda_{w}=\Sigma_{i}^{W} \lambda_{i}$. Thus, the distribution of visits from the aggregate of $W$ individuals is $\operatorname{prob}(Y=n)$ $=\left(e^{-\lambda_{w}}\right)\left(\lambda_{w}\right)^{n} / n !$. This adding-up property facilitates the use of aggregate data, given knowledge of population size.

(b) If a constant term is included in the function describing $\lambda$, the sum (over all observations) of observed demand will equal the sum of predicted demand.

(c) Zero values are admissible. These properties also hold for the negative binomial, with $\lambda$ replaced by $\mu$.

To insure that $\lambda$ (or $\mu$ in the negative binomial) is strictly positive, it is postulated that

$$
\lambda(P, Z ; \beta)=\exp \left(\beta_{0}+\beta_{p} P+\beta_{z} Z\right) .
$$

These count data models are estimated via maximum likelihood (ML) techniques. The Poisson is readily estimated using the Newton-Raphson technique. The negative binomial, especially its Hessian matrix, is more complicated and is usually solved with a quasi-Newton method, such as the BHHH or the DFP algorithms (Judge et al.).

Maximum likelihood estimation assumes that the postulated distribution is indeed correct. This assumption may impose some stringent requirements, such as the $E(Y)=\sigma^{2}(Y)$ criteria of the Poisson. The distributional sensitivity of these models raises concerns about robustness. How badly will these models fail if the true probability distribution deviates from the assumed distribution?

The consequences of these assumptions, and possible means of relaxing them, have been studied by a number of authors. For count data models, the work of Gourieroux, Montfort, and
Trognon is especially useful. ${ }^{4}$ They introduce the concept of pseudo- (PML) and quasi-generalized pseudo (QGPML) maximum likelihood estimation. Basically, they show that if functions describing the true mean and true variance of the dependent variable (say, the number of trips) are known [say, equal to $f(p, z ; \beta)$ and $g(p, z ; \beta, \alpha)$, respectively]; then the Poisson and negative binomial will be consistent, regardless of the underlying distribution. Several interesting results are obtained at the empirical level:

(a) For the Poisson, the estimates of $\beta$ from ML are consistent, and analytically equivalent to the PML estimates. However, the ML estimate of the covariance matrix of $\beta$ is too small. The PML estimator can be used to compute a consistent estimate of the covariance (CV) matrix, which turns out to be a function of the "true variance" [the function $g(P, Z ; \beta, \alpha)$ introduced above].

(b) For the negative binomial, the ML estimate of $\beta$ is not consistent if the distributional assumptions do not hold. A two-step QGPML estimator can be used to consistently estimate both $\alpha$ and $\beta$. In this case, both $\beta$ and the $\mathrm{CV}$ matrix are functions of the true variance.

\section{An Application to the Boundary Waters Canoe Area}

1980 permit data from the Boundary Waters Canoe Area (BWCA) in northern Minnesota will be used to compare the various count models. Each permit contains the ZIP code of the group leader, which can be used to obtain distance-tosite information as well as socioeconomic variables either at the zip code level or at the county level. Because no other information is available, especially information on number of prior trips, the permits must be aggregated. County-level aggregates are used for this analysis. Therefore, the dependent variable is number of trips per county.

A total of 27,433 overnight permits were aggregated into the 1,396 counties within 1,000 road miles of the BWCA. ${ }^{5}$ About half of these

\footnotetext{
${ }^{4}$ Other works in this field include White, who offers a general discussion of the consequences of using an incorrect distribution function, and McCullagh and Nelder, who embed count models in the framework of generalized linear models. An interesting summary of count models as weighted, iterative linear estimators is presented by Hall, Hausman, and Griliches.

${ }^{5}$ All overnight visitors are required to obtain a permit. The entire data base of permits comprises a full census of overnight visitors, who account for about $50 \%$ of BWCA use (in RVD terms).
} 
counties have zero visits. Travel cost was based on $\$ 0.076 /$ mile (1980\$) plus a time cost computed using one-third of the per capita wage rate multiplied by average group size (4.0); the result was an average cost per mile of approximately $\$ 0.20$.

Table 1 contains the results from several models. An exponential form is used, with an individual's expected demand equal to $\exp (X \beta)$, where $X$ is a vector of exogenous variables. Because aggregate data are used, the population of each county must also be considered. For the count models, the adding-up property suggests use of population as a multiplicative weight. Thus, $E\left(Y_{\text {county }}\right)=P O P * \exp \left(X_{\text {county }} \beta\right) ; Y_{\text {county }}$ is the aggregate number of visits from the county, $P O P$ is the population of the county, and $X_{\text {county }}$ is a set of county-level exogenous variables (see table 1 for a description of the exogenous variables). For comparative purposes, a simple per capita semilog model is also estimated. Consumer surplus $(C S)$ estimates (in 1980\$) are computed using equation (2), with equation (3) used for $\lambda^{*}{ }^{6}$ In all models, the reported $C S$ estimates use all 1,396 counties.

The most noticeable results are that the ownprice coefficient $(B W T C)$ is negative and significant for all models. This is especially true for the Poisson model. However, if the Poisson assumption of $E(Y)=\sigma^{2}(Y)$ is incorrect, then the standard errors generated by the ML estimator of the Poisson will also be incorrect. To test this assumption, a score test devised by Lee is computed. This test is normally distributed under the null hypothesis that the Poisson model is correct. The results of this test, and the large value of the $t$-statistic for $\alpha$ in the negative binomial model, indicate that the Poisson is inappropriate [that $E(Y)$ does not equal $\sigma^{2}(Y)$ ]. Thus, the CV matrix computed by the ML estimator is incorrect, suggesting use of the PML estimator for the Poisson model. In the PML estimator, $B W T C$ is still significantly negative, but several of the demand shifters (the age variables, percent unemployment, and percent poverty) are insignificant at the $95 \%$ level.

The negative binomial model returns qualitatively similar coefficients for $B W T C, \% C O L$ -

\footnotetext{
${ }^{6}$ To maintain consistency with the definition of the market area (all counties within 1,000 miles), a choke price equal to the maximum price in the sample is used. Alternatively, a choke price of infinity could be used. However, when $C S$ is computed using a choke price of infinity, the results differ by less than $1 \%$. Note that the market area is limited in order to limit the bias resulting from visitors who partake in multiple-destination trips.
}

$L E G E$, and INCOME. The sign on the substitute price $(A P T C)$ is now positive, the theoretically anticipated sign. Also, the age variables are significant.

Within count models, the effect on consumer surplus is on the order of $25 \%$. A much greater change (50\%-100\%) occurs between count and semilog OLS models. In RVD terms, given an average $C S$ of $\$ 1.5$ million, and a total of 25,000 groups of four individuals spending four days in the wilderness, the average RVD value will be about $\$ 4.00$ (a fairly small value).

The semilog OLS model uses two heuristics: zero observations are dropped when coefficients are computed, and a bias correction factor is computed. The drop zeros rule ensures computability of the model. Since it discards information about nonvisitors, a potential for bias exists. Alternatively, the semilog model could be estimated using a nonlinear maximum likelihood technique (Creel and Loomis). While such an approach permits zero visits, the treatment of demand shocks resulting from fluctuations in the error term (presumably caused by changes in unobservable factors) is not consistent with demand shocks caused by fluctuations in observed exogenous variables; with changes in unobservable factors having an additive impact, changes in observable variables have a proportional impact. Although this feature may or may not be appealing, for purposes of comparison the simpler drop zeros method is adopted.

The bias correction factor (Stynes, Peterson, and Rosenthal) is a simple multiplier guaranteeing that the sum of observed demand equals the sum of predicted demand. It has substantial impact, leading to a doubling of $C S$. Alternatively, Bockstael and Strand argue against inclusion of such a bias correction factor. However, the bias correction factor is used since unbiased estimation of $E(C S)$ requires an unbiased estimate of $E(Y)$.

Formal qualitative comparisons between models are presented in table 2 and by examining the $\eta^{2}$ goodness-of-fit statistic in table $1 .^{7}$ Table 2 displays the results of an out-of-sample test created by Ashley and popularized by Shaw.

\footnotetext{
${ }^{7}$ The $\eta^{2}$ statistic can be described as a measure of the correspondence between observed and predicted values. It is related to the familiar $R^{2}$ statistic. Specifically, $R^{2}=E S S / T S S$ and $\eta^{2}=1-$ $R S S / T S S$, with ESS the explained sum of squares, TSS the observed (total) sum of squares, and $R S S$ the residual sum of squares. In linear models, these two statistics are analytically equivalent, but they may diverge in nonlinear models. For further discussion of goodness of fit statistics in nonlinear models, see Peterson and Stynes.
} 


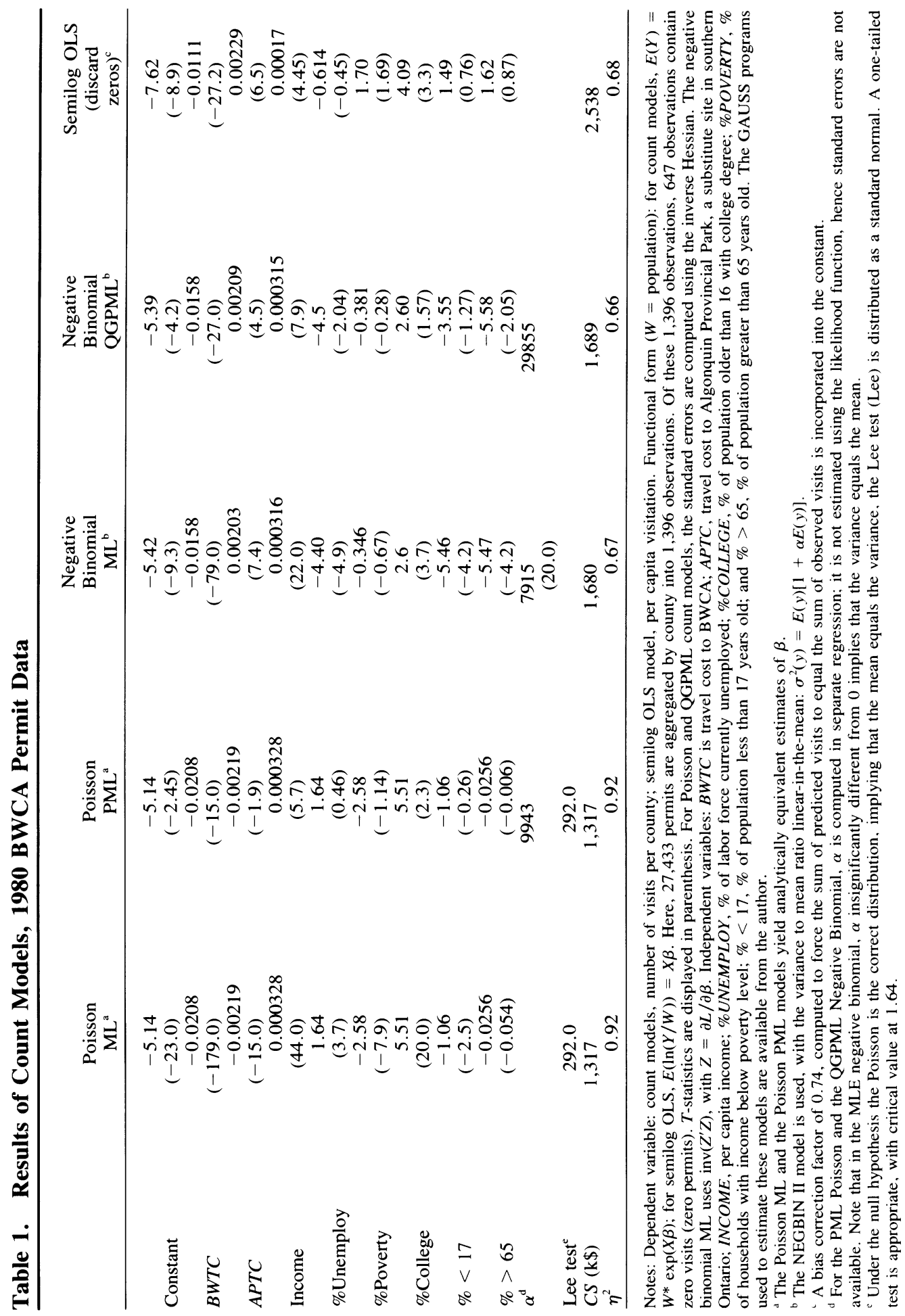


Table 2. Ashley Test of Model Quality

\begin{tabular}{lll}
\hline Model $1^{\mathrm{a}}$ & Model 2 & The superior model is ... \\
\hline Poisson & NegBin & inconclusive \\
Poisson & QGPML & inconclusive \\
Poisson & Semilog+ & Poisson \\
NegBin & QGPML & NegBin \\
NegBin & Semilog+ & NegBin \\
QGPML & Semilog+ & QgPML \\
\hline
\end{tabular}

Note: The Ashley test (Ashley, or Shaw) uses out-of-sample data (1981 data) to directly compare the predictive power of two different models.

${ }^{a}$ Models: Poisson, Poisson model; NegBin, negative binomial estimated using ML; QGPML, negative binomial estimated using QGPML; Semilog+, OLS on semilog model with a "bias correction" factor.

This test is essentially a nonparametric comparison of the goodness of fit of two different models, using out of sample data. In this case, 1981 data are used as the out-of-sample data, and 1980 data are used to estimate the coefficients. ${ }^{8}$

The $\eta^{2}$ goodness-of-fit statistics suggest that fit is fairly good, especially for the Poisson model. The negative binomial have predictive accuracy similar to the bias-corrected semilog. The results of the Ashley test, although not conclusive, suggest that count models are superior to the bias-corrected semilog model. Within count models, the evidence is weaker. For example, the ML negative binomial is considered superior to the QGPML negative binomial. It is interesting that a Hausman specification test (Hausman) comparing the QGPML and the ML estimators fails to reject the consistency of the ML estimator; the result indicates that the negative binomial distribution is correct.

These results indicate that count models outperform the drop-zeros semilog model. Within count models, the Poisson outperforms the negative binomial in predictive accuracy but produces incorrect measures of variability, throwing the Poisson $t$-statistics into doubt. The PML estimator of the Poisson can be used to address this failure while maintaining the predictive power of the Poisson. The QGPML negative binomial is similar to the ML negative binomial, suggesting either that the ML estimator be used (on

\footnotetext{
${ }^{8}$ The Ashley test uses out-of-sample data. To test that 1981 data is out of sample but not generated by a different model, parameter stability is tested. Specifically, a Chow test of parameter equality, using 1980 and 1981 data with a Poisson PML model, failed to reject the null of parameter stability; with an $F(9,2774)$-statistic of 0.92 , well below the $95 \%$ cutoff level at 1.88 . However, the MLE model did reject the null, with an $F$-statistic of 42.0 .
}

efficiency grounds) or that the QGPML estimator be used (on ease of computation grounds).

\section{Concluding Comments}

The intrinsic nature of site visitation suggests that demand models based on continuous functional forms are inappropriate because they fail to recognize the count nature of trip making. To account for this feature, two count models, the Poisson and negative binomial, are reviewed. At an empirical level, besides matching the integer quality of trip demand, these count distributions explicitly account for censoring at zero. Furthermore, a behavioral justification for their use, based on a repeated discrete choice process generating trip demand, can be derived.

An application to permit data from the Boundary Waters Canoe Area reveals that the choice of estimator can have substantial impact, especially on consumer surplus estimates. In particular, the drop-zero semilog OLS yielded estimates approximately $50 \%$ larger than the Poisson. The consumer surplus differences among the several count models were smaller. This suggests that for welfare purposes, the ML Poisson may be adequate. However, coefficient values did vary, especially for demand shift variables. More important, $t$-statistics for all variables were quite different across count models, with the robust estimators (such as the PML Poisson) generally returning much smaller $t$-statistics. These results suggest caution when interpreting coefficient values from maximum likelihood estimators. ${ }^{9}$

Although the application of count models to travel cost analysis is becoming increasingly popular, the existence of robust estimators for both the Poisson and negative binomial has not been exploited. These robust estimators reduce the extent of a priori knowledge required for consistent estimation of the coefficient vector and its covariance matrix.

A discussion of the aggregation issue also is in order. First, consider the robust estimators used here. They all require that the functional form describing expected demand is correct. In the context of aggregate data, this requires that the

\footnotetext{
${ }^{9}$ To the extent that variance of consumer surplus is a function of the covariance of the coefficient vector, this result also suggests caution when qualitative comparisons are formed. See Bockstael and Strand or Adamowicz, Fletcher, and Graham-Tomasi for further discussion of the effects of uncertainty on the variance of consumer surplus estimates.
} 
$X_{\text {county }}$ measures be representative, in the sense of Deaton and Muelbauer (p. 149), of the county. If they are not, then $W^{*} \exp \left(X_{\text {count }} \beta^{*}\right)$ will not equal $\sum_{i=1}^{W} \exp \left(x_{i} \beta^{*}\right)$, and the requirements for robust estimation will be violated.

This weakness of aggregate data sets must be measured against the weaknesses of alternative methods, such as the sample selection models of Shaw or the truncated count models of Creel and Loomis. These models admit the influence of nonvisitors in a limited fashion. First, for tobit-like estimators and for Poisson-based models, incorrect specification of higher moments will bias estimates of demand parameters. Although the negative binomial is robust to misspecification of higher moments (Grogger and Carson), all these models are sensitive to the presumption that nonvisitors possess the same demand parameters as visitors. To the extent that this is not true, truncated models may be more biased than aggregated models. In other words, aggregate models permit nonvisitors to influence estimation, so that the resulting parameters are a reduced form incorporating information on both visitors and nonvisitors. For many purposes, such as calculating the $C S$ for a new population, such parameters may be superior to those produced by truncated models. ${ }^{10}$ In short, aggregate analysis is not necessarily dominated by site-based samples estimated with econometric techniques that recognize truncation.

\section{[Received April 1990; final revision received August 1990.]}

\section{References}

Adamowicz, Wiktor, Jerald Fletcher, and Theodore Graham-Tomasi. "Functional Form and the Statistical Properties of Welfare Measures.” Amer. J. Agr. Econ. 69(1989):414-21.

Ashley, R., C. W. J. Granger, and R. Schmalensee. "Advertising and Aggregate Consumption: An Analysis of Causality." Econometrica 48(1980):997-1016.

Bockstael, Nancy, and Ivar Strand. "The Effect of Common Sources of Regression Error on Benefit Estimates." Land Econ. 63(1987):11-20.

Cameron, Colin, and Pravin Trivedi. "Econometric Models Based on Count Data: Comparisons and Applications of Some Estimators and Tests." J. Appl. Econometrics 1(1986):29-53.

\footnotetext{
${ }^{10}$ Furthermore, extensions to the Poisson that permit systematic differences between visitors and nonvisitors, such as proposed by Mullahy, are estimable with nontruncated samples (although the robustness of these models requires further investigation).
}

Creel, Michael, and John Loomis. "Theoretical and Empirical Advantages of Truncated Count Data Estimators for Analysis of Deer Hunting in California." Amer. J. Agr. Econ. 72(1990):434-45.

Deaton, A., and J. Muelbauer. Economics and Consumer Behavior. New York: Cambridge University Press, 1980.

Gourieroux, C., A. Monfort, and A. Trognon. "Pseudo Maximum Likelihood Methods: Applications." Econometrica 52 (1984):701-20.

Grogger, J. T., and R. T. Carson. "Models for Counts from Choice Based Samples.” Dep. Econ. work. pap., University of California, July 1988

Hall, Bronwyn, Jerry Hausman, and Zvi Griliches. "Patents and R\&D: Is There a Lag?" Int. Econ. Rev. 27(1986):265-82.

Hausman, Jerry. "Specifiation Tests on Econometrics." Econometrica 46(1978):1251-70.

Hausman, Jerry, Bronwyn Hall, and Zvi Griliches. "Econometric Models for Count Data with an Application to the R\&D Relationship." Econometrica 52(1984):909-37.

Hellerstein, Daniel, and Robert Mendelsohn. "Modeling Recreational Demand as a Repeated Discrete Choice Process: The Case for Count Models." Washington DC: U.S. Department of Agriculture, Econ. Res. Serv. work. pap.

Judge, G., W. Griffiths, R. Hill, H. Lütkepohl, and T. Lee. The Theory and Practice of Econometrics. New York: John Wiley \& Sons, 1980.

Lee, Lung-Fei. "Specification Test for Poisson Regression Models.” Int. Econ. Rev. 27 (1986):689-706.

Maddala, G. S. Limited-Dependent and Qualitative Variables in Econometrics. Cambridge: Cambridge University Press, 1983.

Mood, Alexander, Franklin Graybill, and Duane Boes. Introduction to the Theory of Statistics. New York: McGraw-Hill Publishing Co., 1974.

Mullahy, John. "Specification and Testing of Some Modified Count Data Models." J. Econometrics 33(1986):341-65.

McCullagh, P., and J. A. Nelder. Generalised Linear Models. London: Chapman and Hill, 1983.

Peterson, George, and Daniel Stynes. "Evaluating Goodness of Fit in Nonlinear Recreation Demand Models." Leisure Sci. 8(1986):131-47.

Shaw, Dai Gee. “On-Site Sample's Regression: Problems of Non-Negative Integers, Truncation, and Endogenous Selection.” J. Econometrics 37(1988):211-23.

Small, Kenneth, and Harvey Rosen. "Applied Welfare Economics with Discrete Choice Models." Econometrica 49(1981): 105-30.

Smith, V. Kerry. "Selection and Recreation Demand." Amer. J. Agr. Econ. 70(1988):29-36.

Stynes, Daniel, George Peterson, and Donald Rosenthal. "Log Transformation Bias in Estimating Travel Cost Models." Land Econ. 62(1986):94-103.

Ziemer, Rod, Wesley Musser, and R. Carter Hill. "Recreation Demand Equations: Functional Form and Consumer Surplus.” Amer. J. Agr. Econ. 62(1980):13641 . 